\title{
VA-ADD - VEHICLE AND MOBILE ASSISTED DATA DELIVERY IN CLUSTER BASED VEHICULAR ADHOC NETWORKS
}

\author{
M. Abhilash, K. Lohitha and K. Patya \\ Department of Computer Science and Engineering, Sri Venkateswara Engineering College for Women, India
}

\begin{abstract}
Exciting features like on-demand video streaming in vehicles, profound traffic analysis and automatic flooding of emergency, safety measures from vehicles attracts researchers to dig out more innovations in the field of intelligent transportation system (ITS). Therefore, VANET's plays major role in development of ITS. Such applications require highly reliable communication paths and stable cooperative nodes to support high quality data delivery within expected time. Earlier, a lot of research has been carried out in this area using network formed by vehicles alone and efficiently handles path construction process. But, in certain situations like when the cluster head not able to find an immediate neighbor for forwarding critical information through network might causes inconvenience, also chances of great damage for vehicles along the path. To address this issue in our proposed model, both vehicles and mobile nodes is used dynamically to establish a path depending on situation. And analysis shows there is a significant part of improvement in network performance in aspects of throughput and packet delivery ratio.
\end{abstract}

Keywords:

VANET, MANET, Routing, Markov Process, Clustering, Path Reliability

\section{INTRODUCTION}

Vehicular ad hoc networks (VANET's) play a vital role in development of intelligent transportation system (ITS) because of some important reasons given here: Due to the rapid growth of vehicles on the roads increases traffic control and maintenance issues, unexpected delays and pollution [1]. Another important point is expectations of users in vehicles are vastly increasing day by day, they want everything should done on hands like entertainment services, instant traffic information, on-line bill payments and toll pay etc. Apart from this, the safety services like collision warning, lane changing messages are predominantly required. So, possibly VANET's is method solution to handle all the above services. In VANET's network is formed by the group of vehicles in which every vehicle is going to acts as sender and receiver for packets transmission between source and destination using the DSRC (Dedicated short-range control) protocol which gears up to $300 \mathrm{~m}$ range [2]. In general, communication happens between vehicles to vehicle $(\mathrm{V} 2 \mathrm{~V})$ and vehicles to infrastructure (V2I) as shown in Fig.1 with VANET's frequent changes in network topology causes path disruption as a consequence packet loss, delay will occur. Hence it is required to maintain reliable path between source and destination by choosing perfect nodes while packets forwarding. So, routing protocols should dynamically analyze the situation and adjust paths accordingly using intermediate nodes.

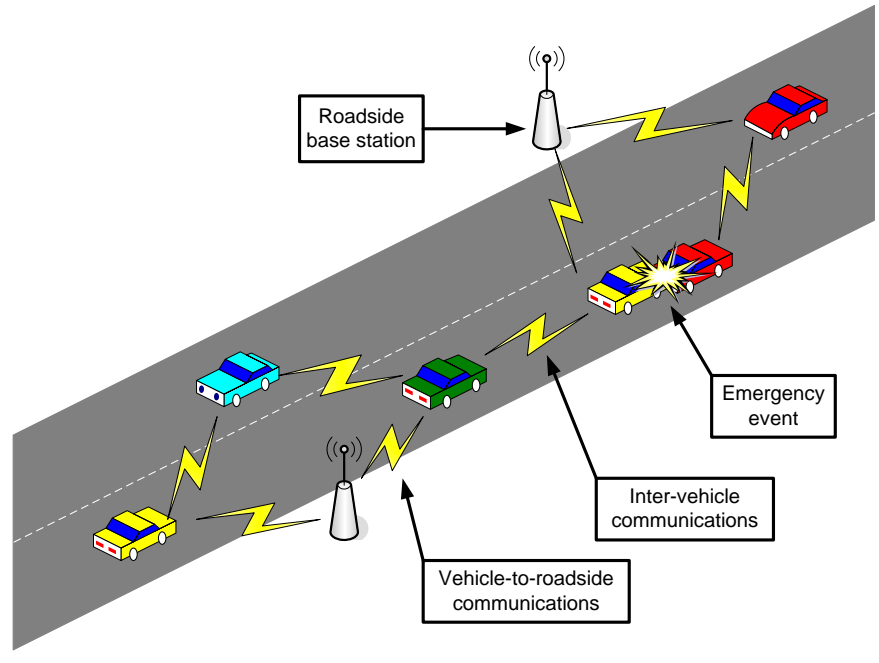

Fig.1. VANET Architecture

Existing routing protocols [3] used topology information, i.e. Link information between nodes to carry and forward to the specified destination by seeing link status information network chooses competent nodes to forward data. In proactive [4] routing chooses the shortest path between the source and destination using information like speed, direction and position that is stored in tables. Fisheye state routing [5] is an example for PRP. In this technique to update the link status, each node sends information to only neighbor nodes. So that network overhead is reduced, but complexity increases with the size of networks. The drawback of using PRP is wastage of bandwidth because only a few paths would be used for route construction. Ad hoc on demand distance vector routing protocol (AODV) [6] also called as the reactive routing protocol in which route is established on demand only. Neighbor node status can gather by broadcasting hello messages, on receiving positive acknowledgement the node forwards packets, process repeats up to the point of destination node. DTN [7] used carry and forward technique when forwarded cannot see the neighbor node and GPSR [8] protocol selects nearby nodes towards a destination and it uses beacons to find the node with a short distance. Above discussed protocols use only vehicle network for routing process. Logically speaking VANET's are sub part of MANETs [9] this feature motivates to design proposed system which combines vehicle and mobile devices for routing process. "Generally, in every moving vehicle we can expect a minimum of one or two mobile devices."

\section{LITERATURE SURVEY}

In Dynamic source routing [10] source node search for a path (route) i.e. route discovery process will start by asking neighbors to know about the destination and data sent through RREQ (route 
request) packet. Every packet given with unique IDS, list of intermediate nodes, source node and the destination node. In broadcasting process, if the sender receives duplicate packets simply it drops, detected path details going to store in the cache for future references, this is called as route caching. If a link is broken or node detachments occur, then the problem arises, that will be solved by route maintenance by sending RERR (route error packet), then the detected route will be removed from the cache. VANET is a subset of mobile ad-hoc networks. It is a recently presented innovation. VANET enables vehicles to communicate with each other and share information in a wireless network if the vehicles are inside the range. VANET has some different characteristics than the MANET that makes it unique. There can be two types of VANET V2V - when there is no infrastructure required, nodes do vehicle to vehicle communication with each other V2RSU - when rode side units are used for routing information exchange with alternate vehicles.

In Greedy perimeter stateless routing [11] every node broadcast its IP address, position periodically with neighbors and selects nearer nodes as intermediate nodes. In distance vector routing [12] every node maintains a table, maintains distance between nodes and continuously exchanges link status table with their neighbors.

A Contention-Based Protocol (CBP) [13] used for operating wireless telecommunication as the communications protocol, here without pre-coordination protocol equipment will allow many users to use the same radio channel. The "listen before talk" operating procedure is the most well-known contention-based protocol. A protocol that allows multiple users to share the same spectrum by defining the events that must occur when two or more transmitters attempts to simultaneously access the same channel and establishing rules by which a transmitter provides reasonable opportunities for other transmitters to operate. A vehicular network, many delivery applications can be supported without extra hardware cost. However, existing protocols are not suitable for supporting delay tolerant application in sparsely connected vehicular networks. To address this problem, the idea of helper node is opted, where a moving vehicle carries the packet until a new vehicle moves into its vicinity and forwards the packet.

GeOpps [14] uses GPS and digital maps for establishing routes; here navigation system provides nodes and path information throughout the path. A node with the minimum arrival time selected to forward packets.

When a vehicle issues a delay-tolerant data query to some fixed site, the techniques to efficiently route the packet to that site and receive the reply within a reasonable delay is occurred. To overcome it, CAR [15] is an efficient protocol also suitable for highway and city environments, here AODV technique used for path discovery process and based on connection awareness only forwards packets to nearby nodes.

VADD (Vehicle Assisted Data Delivery) [16] used to carry and forward packets in moving nodes until a carrying packets floods hello messages along the path to find nodes on reply it forwards to a particular node.

\section{PROPOSED METHOD}

A vehicular network, many delivery applications can be supported without extra hardware cost. However, existing protocols are not suitable for supporting delay tolerant application in sparsely connected vehicular networks. In Vehicle-Mobile assisted data delivery (VM-ADD) initially vehicles on roads form a group of clusters in which both vehicles and mobile nodes are utilized for forwarding packets when the cluster head cannot figure out intermediate nodes due to unavailability of ready nodes, to continue packets forwarding process proposed system used mobile nodes available in the vehicle. In proposed model, vehicle and mobile assisted data delivery technique cluster head picks either vehicle or mobile node based on available information maintained by status tables for making forward decision as shown below.

Vehicles Information Table
\begin{tabular}{|l|l|l|l|}
\hline Vehicle id & Position & Status & direction \\
\hline & & & \\
\hline Mobile Nodes information table \\
\hline Mobile id & Vehicle Id & Status & direction \\
\hline & & & \\
\hline
\end{tabular}

Fig.2. Tables Maintained by $\mathrm{CH}$ (Vehicles and Mobiles)

Assuming mobile devices situated in vehicles also moves with same speed and direction. The proposed method is whenever $\mathrm{CH}$ will not able identify neighboring node (free node) in cluster region instead of searching vehicle node, it chooses mobile nodes to carry and forward packets (temporary storage) and once vehicle link gets up packets are handover to vehicle node.

\section{PROPOSED FRAMEWORK}

\subsection{CLUSTER FORMATION}

The proposed model uses clustering technique [17] where vehicles on roads divided into groups based on geographical area. Selection of Cluster head $(\mathrm{CH})$ is based on the maximum time (staying of that node in that region), speed, and distance. That elected cluster head will maintain the information about remaining nodes (cluster members) which moves in the same regions. $\mathrm{CH}$ maintains table consists of both vehicle and mobile nodes information like speed, distance, status, direction and time of all nodes in the cluster. Whenever $\mathrm{CH}$ moves out of the region immediately table information will move to the next selected $\mathrm{CH}$.

\subsection{PATH SELECTION PROCESS}

Scenario-1: Whenever the source requests for data transfer the cluster head $(\mathrm{CH})$ verifies all eligible nodes in its region on parameters like position, current load and, link status. In VANET's position information can be obtained with global position System (GPS) and node status information calculated using Bernoulli Trail (BT) method shown in the next section. Initially $\mathrm{CH}$ sends hello messages to neighbor nodes BT method tags, nodes with Success (S) or Failure (F) based on probability. For e.g. if the $\mathrm{CH}$ sends 5 hello messages to target nodes and receives 3 responses in stipulated time (response count $>3$ ) such nodes are selected as next forwarder nodes for packet transmission.

Scenario-2: In typical situations like, if $\mathrm{CH}$ not able to see ready forwarder(vehicle) during of vehicle node (success) in such 
case proposed method efficiently shifts control in search of available mobile nodes, here both Bernoulli Trail (BT) and Markov process used for taking forward decision i.e. Either to forward packets towards mobile node or vehicle node. BT helps in providing current status information of mobile and vehicle nodes based on probability of success Markov process will make forwarding decisions.

Scenario-3: In this case, when $\mathrm{CH}$ looking for intermediate nodes first it verifies whether there is availability of unused bandwidth in the same direction of current path. If $\mathrm{CH}$ finds BW availability temporarily forward packets along the path until it finds new node (vehicle). If utilization of bandwidth revoked by earlier assigned nodes, then the packets will be handover to the nodes at that corresponding region. So that it can avoid resending packets process. In proposed forwarding packets to the intermediate nodes clearly logical and efficient, depends on situation and based on probability of success ratio i.e. Markov decision process for data forwarding and Bernoulli trail technique for selecting reliable nodes.

\section{METHODOLOGY}

Markov Process model and Bernoulli Trail [18] methods are discussed and efficiently applied for selecting intermediate nodes during path construction process:

\subsection{MARKOV PROCESS}

A mathematical system [19] having finite or countable number of possible states that undergoes transmission between one state to another based on the probability. Markov process is a memoryless random process i.e., the next move or state transmission will always depend on the present state of the node and it does not have any method about previous states. This is called as Markov property.

A Markov chain can be described as a set of states, $S=\left\{s_{1}\right.$, $\left.s_{2}, \ldots, s_{r}\right\}$, where the process starts in one of the states and moves successively from one state to another, where each move is called a step. The changes of state of the system are known as transitions. Hence, probabilities related to these various state-changes are known as transition probabilities. For example, if the chain is currently in state $s_{i}$ then it moves to state $s_{j}$. The probability of moving from one step to another is indicated by $p_{i j}$. The set of all states and transition probabilities completely characterizes a Markov chain.

This probability does not depend upon which states the chain was in before the current. With random changing systems, it will be difficult to predict with certainty the state of a Markov chain at a given point in the future. However, the statistical properties of the system's future can be predicted. In many applications, it is these statistical properties that are important.

A Markov process is a stochastic process that has no memory. That is, the probability that the system moves into a particular state depends only upon the state it is currently in, and not on the history of the past visitations of states. Thus, a Markov process can be fully specified via a set of transition probabilities $\pi_{i j}$ that describe the likelihood that the system moves into state $j$ given that it is presently in state $i$. The full set of transition probabilities can be viewed as a matrix $\pi$.
As a simple example, we can consider a system that can occupy any of three states. The probability of moving from one state to another in a Markov process is given via the transition probability matrix (TPM)

$$
\Pi \equiv\left(\begin{array}{lll}
\pi_{11} & \pi_{12} & \pi_{13} \\
\pi_{21} & \pi_{22} & \pi_{23} \\
\pi_{31} & \pi_{32} & \pi_{33}
\end{array}\right)=\left(\begin{array}{lll}
0.1 & 0.5 & 0.4 \\
0.9 & 0.1 & 0.0 \\
0.3 & 0.3 & 0.4
\end{array}\right)
$$

Now consider what happens in a process that moves from one state to another, each time selecting the new state according to the transition probabilities given here. One could construct a histogram to describe the number of times visited in each of the three states during the process. After a long period of sampling, steady state is reached and the histogram does not change with continued sampling. The histogram so obtained is called the limiting distribution of the Markov process. Examine the applet in Illustration 1 to see Markov sampling in action.

So what is the connection to Monte Carlo integration? The scheme is to devise a Markov process to yield a limiting distribution that covers the important regions of our simulated system. In this manner we can do importance sampling of a complex region of integration by specifying only the transition probabilities of the Markov process. To accomplish this we need to develop the connection between the transition probabilities and the limiting distribution.

Several important features should be noted: first, each probability is properly specified, i.e. it is nonnegative and does not exceed unity; second, each row sums to unity, indicating a unit probability for going from one state to another in a given step; third, the diagonal elements are not necessarily zero, indicating that an acceptable outcome for a Markov step leaves the system in its present state. More on this detail later. In all of what follows it is important that we have a transition-probability matrix that corresponds to an Ergodic process. Ergodicity was discussed in a previous section. In this context, it means that it is possible to get from one state to another via a sufficiently long Markov chain.

The utility of detailed balance is not in determining the limiting distribution from a set of transition probabilities. In fact, our need is the opposite: we have a specification for the distribution of states, and we want to apply a Markov process to generate states according to this distribution; how can we construct appropriate transition probabilities to achieve this aim? As demonstrated in Illustration 2, there are many possible choices for the set of transition probabilities that yield a given limiting distribution. Of course, we need to generate only one set to perform the Markov process. The choice of transition probabilities can be dictated by convenience and performance (that is, how well they sample all relevant states for a finite-length Markov chain).

Detailed balance is an extremely very useful guiding principle, because it leads us to generate a valid set of transition probabilities while considering them only a pair at a time. This contrasts with the full eigenvector equation, which involves all the states at once. The implication there is that all of the transition probabilities must be specified together and at one time, so to satisfy this relation between them. By focusing instead on the sufficient condition of detailed balance, a great burden is removed. We do not have to evaluate all transition probabilities for all states at once, and in fact we do not have to evaluate all transition probabilities, period. 
Instead we can get away with evaluating them only as we need them. The calculation of the transition probabilities can be tied right into the Markov chain, so that only those encountered during a given sequence are actually computed. The number of microstates in a typical statistical mechanics system is huge, so there is immense savings in exploiting this "just-in-time" calculation scheme given to us by detailed balance. Still, one should not lose sight of the fact the microscopic reversibility is not required, and that it may be advantageous to violate the principle at some times; but one should take caution that alternative transition probabilities are consistent with the expected limiting distribution. Oddly enough, in a molecular simulation (and unlike the simple examples given here) there is no easy way to check, even some posteriori, that the limiting distribution generated by the Markov sequence actually coincides with the target distribution. Consequently, it is quite possible for an error in the formulation of the transition probabilities to remain undetected.

Markov process can be represented either in directed graph or in matrix form. Consider, three nodes. The probability for each node can be calculated by using Bernoulli probability as shown in Fig.3.

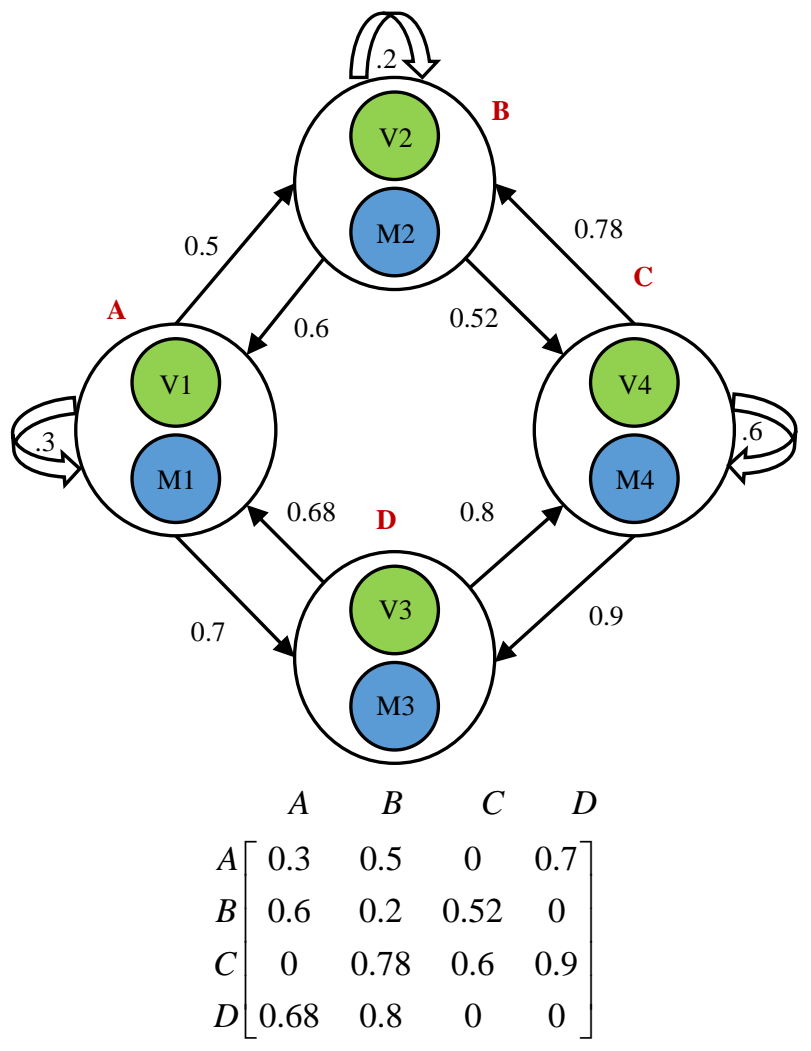

Fig.3. Markov Decision Process and matrix values

\subsection{BERNOULLI TRAIL METHOD}

Bernoulli Trail used in our proposed work is used to find stable nodes on repeated trials (flooding hello messages). BT comes up with exactly two possible outcomes "success" or "failure" the probability of success and the probability of failure sum to unity (one), since these are complementary events: "success" and "failure" are mutually exclusive and exhaustive as shown in Eq.(2).

$$
P(k)=\left(\begin{array}{l}
n \\
k
\end{array}\right) p^{k} q^{n-k}
$$

where, $n=$ number of times hello messages sent for each vehicle, $k=$ number of success vehicles, $n-k=$ number of failure vehicles, $p=$ probability of success in one trail of vehicles and $q=1-p=$ failure probability in one trail.

A Bernoulli process is considered as $X=\left\{X_{n} ; n=1,2 \ldots.\right\}$ where each $X$, is a Vehicle set, based on traffic conditions count may varies and tags nodes with $S$ or $F$ based on probability. Now applying Markov decision process for taking forward decisions, suppose that $Y=\left\{Y_{n} ; n \geq 1\right\}$ is a Markov chain with some transition matrix $P$ on a discrete state space $E$. Moreover, $v(i)=$ $P\left[Y_{1}=i\right]$ represents the initial set if vehicles of $Y$.

$$
p\left[X_{1}=x_{1}, X_{2}=x_{2}, \ldots, X_{n}=x_{n} \mid Y\right]=\prod_{k=1}^{\pi} P\left[X_{k}=x_{k} \mid Y\right]
$$

The Eq.(3) uses BT results and analyses neighbour nodes success and failure tags and forwards packets to most reliable neighbour node.

\section{PERFORMANCE ANALYSIS}

The Fig. 4 shows that significant improvement in terms of throughput when number of nodes increases performance also increases which in turn packet loss ratio is also reduced.

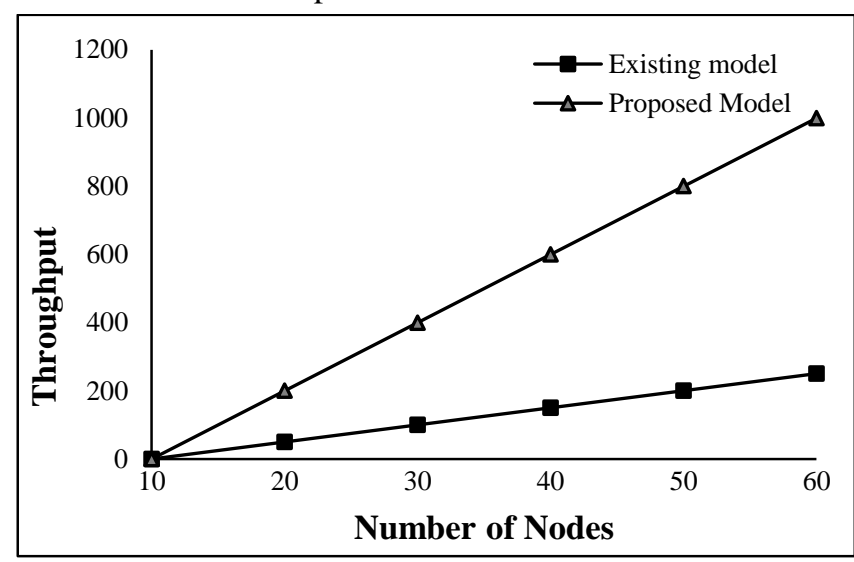

Fig.4. Throughput Analysis

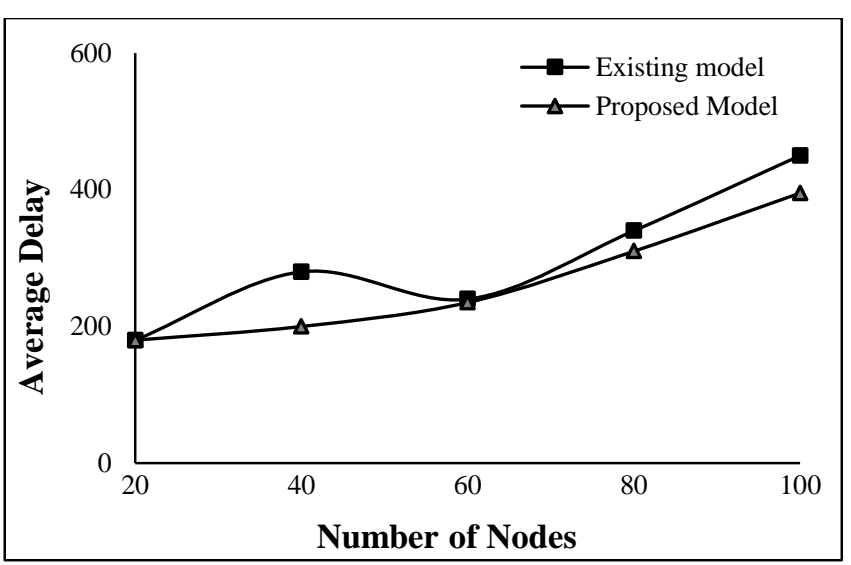

Fig.5. Delay Analysis 
The Fig.5 shows that proposed system constructs reliable paths using competent cooperative nodes so that number of path breaks minimized which in turn reduces delay and improves overall network performance

\section{CONCLUSION}

The performance of VANET is measured to find how efficiently the packets are routed between source and destination. In this proposed work depends on situation VM-ADD technique dynamically chooses intermediate nodes either vehicle or mobile node as intermediate node without delay. Analysis shows performance of VM-ADD technique improves in terms packet delivery ratio and throughput parameters.

\section{REFERENCES}

[1] V.V. Duduku, A. Chekima, F. Wong and J.A. Dargham, "A Study on Vehicular Adhoc Networks", Proceedings of IEEE International Conference on Artificial Intelligence, Modelling and Simulation, pp. 1-4, 2015.

[2] ETSI, "Intelligent Transport Systems (ITS); Framework for Public Mobile Networks in Cooperative ITS (C-ITS)", Available

https://www.etsi.org/deliver/etsi_tr/102900_102999/10296 2/01.01.01_60/tr_102962v010101p.pdf

[3] M. Altayeb and I. Mahgoub, "A Survey on VANETs Routing Protocols", International Journal of Innovation and Applied Studies, Vol. 3, No. 3, pp. 829-846, 2013.

[4] P. Rani, N. Sharma and P.K. Singh, "Performance Comparisons of VANET Routing Protocols", Proceedings of IEEE International Conference on Wireless Communications, Networking and Mobile Computing, pp. 23-28, 2011.

[5] G. Pei, M. Gerla and T.W. Chen, "Fisheye State Routing in Mobile Ad Hoc Networks", Proceedings of IEEE International Conference on Communications, pp. 1-8, 2000.

[6] C.E. Perkins and E.M. Royer, "Ad Hoc On Demand Distance Vector Routing", Proceedings of $2^{\text {nd }}$ IEEE Workshop on Mobile Computing Systems and Applications, pp. 25-29, 2003.
[7] S.M. Tornell, C.T. Calafate, J.C. Cano and P. Manzoni, "DTN Protocol for Vehicular Networks: An Application Oriented Overview", IEEE Communications Surveys and Tutorials, Vol. 17, No. 2, pp. 868-887, 2014.

[8] B. Karp and H.T. Kung, "GPSR: Greedy Perimeter Stateless Routing for Wireless Networks", Proceedings of $6^{\text {th }}$ Annual International Conference on Mobile Computing and Networking, pp. 243-254, 2000.

[9] P. Ranjan and K.K. Ahirwar, "Comparative Study of VANET and MANET Routing Protocols", Proceedings of IEEE International Conference on Advanced Computing and Communication Technologies, pp. 1-7, 2011.

[10] F. Li and Y. Wang, "Routing in Vehicular Ad Hoc Networks: A Survey", IEEE Vehicular Technology Magazine, Vol. 2, No. 2, pp. 12-22, 2007.

[11] P. Fazio, F.D. Rango, C. Sottile, P. Manzoni and C. Calafate, "A Distance Vector Routing Protocol for VANET Environment with Dynamic Frequency Assignment", Proceedings of IEEE International Conference on Wireless Communications and Networking, pp. 28-31, 2011.

[12] S. Ozekici, "Markov Modulated Bernoulli Process", Mathematical Methods of Operations Research, Vol. 45, No. 3, pp. 311-324, 1997.

[13] Miguel Rios, "GeOpps-N: Opportunistic Routing for VANET in a Public Transit System", IEEE Latin America Transactions, Vol. 14, No. 4, pp. 1630-1637, 2016.

[14] V. Naumov and T.R. Gross, "Connectivity-Aware Routing (CAR) in Vehicular Ad-hoc Networks", Proceedings of $26^{\text {th }}$ IEEE International Conference on Computer Communications, pp. 1-5, 2007.

[15] J. Zhao and G. Cao, "Vehicle-Assisted Data Delivery in Vehicular Ad Hoc Networks", IEEE Transactions on Vehicular Technology, Vol. 57, No. 3, pp. 1910-1922, 2008.

[16] S. Jalavandi and R. Rafeh, "A Cluster-based Routing Algorithm for VANET", Proceedings of IEEE International Conference on Computer and Communications, pp. 14-17, 2016.

[17] O.C. Ibe, "Markov Processes for Stochastic Modelling", Academic Press, 2009.

[18] Bernoulli's principle, Available at: https://en.wikipedia.org/wiki/Bernoulli\%27s_principle 